\title{
Monte Carlo and large angle gluon radiation
}

\author{
Yu.L. Dokshitzer ${ }^{1,3 *}$ and G. Marchesini ${ }^{2}$ \\ ${ }^{1}$ LPTHE, Universities of Paris-VI and VII and CNRS, Paris, France \\ ${ }^{2}$ University of Milan-Bicocca and INFN Sezione di Milano-Bicocca, Milan, \\ Italy \\ ${ }^{3}$ CERN Theory Division, Geneva, Switzerland
}

\begin{abstract}
We discuss the problem of incorporating recoil effects into the probabilistic QCD evolution scheme based on the picture of colour dipoles as done in recent Monte Carlo programs. Such a scheme correctly describes subleading soft contributions to multiplicity distributions. However we find that a simple receipt for incorporating recoil effects into the dipole multiplication picture based on the ordering of gluon energies conflicts the collinear factorization and does not lead to the correct DGLAP equation.
\end{abstract}

*On leave of absence: St. Petersburg Nuclear Physics Institute, 188350, Gatchina, Russia 


\section{Contents}

1. Introduction 2

2. Multiple soft gluons and Monte Carlo

2.1 Generating functional in the soft limit 4

2.2 Evolution equation 5

2.2.1 Energy as an evolution variable 6

2.2.2 Integral evolution equation and Monte Carlo 6

2.3 Mean multiplicity 8

2.3.1 The main term 9

2.3.2 The correction term 10

2.3.3 Other observables sensitive to large-angle gluon effects 11

3. Attempt to include recoil in dipole multiplication 11

3.1 Recoil strategy 12

3.2 The non-singlet quark energy distribution 14

3.2.1 Collinear approximation 14

3.2.2 Evolution equation with dipole recoil strategy 15

3.2.3 Analysis of the equation 16

3.2.4 Two gluon emission 18

4. Conclusions 21

A. Global recoil and correspondence with DGLAP 23

\section{Preface}

We share opinion of our late friend Bo Anderson who once bitterly complained about two aspects of scientific publishing that became standard in recent decades: 1) shying away from expressing emotions and 2) not making public an outcome of a study that produced a negative, undesired results. The present paper is moderately emotional; at the same time it is perfectly in line with the second Bo's demand: it reports the finding that the authors did not plan, and would rather not want, to arrive at.

The answer to the question that is posed in this paper suggests that a formulation of Monte Carlo event generation based on the multiplication picture of energy ordered colour dipoles is plugged by a serious problem. 


\section{Introduction}

Generation of events using Monte Carlo methods is an indispensable tool for planning, running and analysing the results of modern high energy experiments. A possibility to generate multi-particle production as a Markov chain of successive independent parton splittings is based on the general property of factorization of collinear singularities which is characteristic for logarithmic field theories (quantum field theories with dimensionless coupling).

Event generation is necessarily an approximate procedure. Strictly speaking, in order to predict the cross section $d \sigma_{n}$ for production of $n$ partons in a hard interaction with the hardness scale $Q^{2}$, one needs to plug in the QCD matrix element of the (at least) $n^{\text {th }}$ order of the perturbation theory. Instead, one repeats $n$ times the basic $1 \rightarrow 2$ splitting process of the first order in $\alpha_{s}$. By so doing one correctly samples the major part of the $n$-parton phase space, though not all of it. It is this part that gives the dominant, maximally collinear enhanced contribution to, e.g., the inclusive particle distribution, $D^{(n)}=\mathcal{O}\left(\alpha_{s}^{n} \log ^{n} Q^{2}\right)$. This approximation known as leading logarithmic (LLA) can be systematically improved by including higher order parton splitting processes. Thus, the next-to-leading logarithmic accuracy corresponding to the series $\alpha_{s}^{n+1} \log ^{n} Q^{2}$ is achieved by incorporating $1 \rightarrow 3$ parton splittings, etc. For inclusive parton distributions this leads to the DGLAP evolution equations [1] whose generalisation to multi-particle distributions can be achieved in the spirit of jet-calculus [2], with additional account of soft gluon coherence.

Beyond the one loop "evolution Hamiltonian" it starts to matter how one organises the parton chain, that is, what one chooses as "parton evolution time". In particular, due to the presence of soft gluons one encounters corrections of the type $\left(\alpha_{s} \log ^{2} x\right)^{k}$ which are formally subleading (non-collinear) but become explosively large in the $x \ll 1$ limit (here $x$ is the gluon energy fraction). This eventuality can be cured by an appropriate tuning of the evolution time parameter. Namely, for time-like parton multiplication (jets) one has to choose the angular ordering [3]. This choice corrects the "naive" fluctuation time ordering (dictated by an examination of Feynman denominators in a cascading process) by taking into clever account collective effects leading to destructive interference in soft gluon radiation (for more details see, e.g., $[4-8])$.

An alternative way of dealing with soft gluon interference effects for time-like parton multiplication (jets) is provided by the "dipole scheme" $[4,9]$ in which an independently radiating parton, $1 \rightarrow 2$, is replaced by a colourless dipole formed by two partons neighbouring in the colour space, $2 \rightarrow 3$. Gluon radiation off a dipole is automatically suppressed at angles exceeding the dipole opening angle thus reproducing the angular ordering. Colour suppressed contributions $\mathcal{O}\left(1 / N_{c}^{2}\right)$ that lie beyond the scope of the dipole approach are numerically small and difficult to trigger experimentally [10]. 
Within the standard coherent parton cascade picture $[5,8]$ it is the small-angle multiplication processes populating jets that enjoy full all-order treatment (get "exponentiated"). The dipole formulation offers a possibility to improve this treatment by taking into consideration logarithmically enhanced effects due to multiple emission of soft gluons at large angles with respect to jets. Non-collinear soft gluons dominate inter-jet particles flows in various hard processes. They also complicate the analysis of the so-called nonglobal QCD observables [11-13], i.e. in observables in which recorded radiation is confined in geometrically definite phase space regions. It is then interesting to involve these corrections into a Monte Carlo, code based on dipole emission, including soft radiation awy from jets.

In this paper we discuss a dipole based scheme (in the large- $N_{c}$ approximation) which is well suited for deriving improved analytic predictions for observables like mean multiplicities, inclusive soft particle spectra and energy flows, correlations, etc., that incorporate large-angle soft gluon radiation effects as in [11-13]. At the same time, we observe that once one aims at beyond the no-recoil (soft) approximation, treating colour dipoles as independently evolving entities is likely to conflict the collinear factorization. It does not lead to the correct DGLAP equation.

The paper is organised as follows. We start by constructing in Section 2 an evolution equation for the generating functional that describes soft gluon content of $e^{+} e^{-}$annihilation events. No recoil is included. This equation is based on the factorization of the multi-soft gluon distribution, in the planar limit, and uses the centre-of-mass energy as an "evolution time". Contrary to what is done normally, no angular variable enters the evolution time here. The evolution equation in the integral form we interpret as a Markov process of successive dipole branchings, which interpretation leads to a Monte Carlo chain process for probabilistic event generation. As a first example, we solve the evolution equation for mean gluon multiplicity to analyse subleading corrections due to large-angle soft gluon radiation. Here we work in the soft approximation meaning: 1) small energies of emitted gluons and 2) neglecting the energy-momentum loss (recoil) by the primaries.

These two aspects of the "soft gluon approximation" do not necessarily go together. In Section 3 we consider an inclusive energy distribution of the leading quark in the quasi-elastic limit, $(1-x) \ll 1$, where emitted gluons ought to be soft but the quark recoil is essential. At the beginning, in the construction of the evolution equation no recoil effects were included. They are incorporated later by using the recoil strategy that seems natural for a dipole multiplication scheme, see [14]. We find, however, that the quark fragmentation function so obtained deviates essentially from the collinear resummation result given by the DGLAP evolution equation. This is in contrast to what is happening in Monte Carlo schemes based on angular ordered time-like cascades where collinear singularities are correctly resummed $[5,15]$.

Discussion of the problem encountered is relegated to the Conclusions section. 


\section{Multiple soft gluons and Monte Carlo}

The method of generating functionals makes it straightforward to generate exclusive events using Monte Carlo techniques. We start from the known $n$-gluon distribution in the soft limit and construct the evolution equation for the corresponding generating functional.

The generating functional for a quark-antiquark pair $q \bar{q}$ plus an ensemble of $n$ secondary partons, $\gamma^{*} \rightarrow p_{a} p_{b} q_{1}, q_{2}, \ldots q_{n}$, is

$$
G(Q, u)=\sum_{n=0}^{\infty} \int d \Phi_{a b}^{(n)}\left(q_{1}, q_{2}, \ldots, q_{n}\right) u_{a} u_{b} \prod_{i=1}^{n} u\left(q_{i}\right)\left|M_{a b}^{(n)}\left(q_{1}, \ldots, q_{n}\right)\right|^{2} .
$$

Here $p_{a}, p_{b}$ are the final on-mass-shell momenta of the quark and antiquark produced by the virtual photon $\gamma^{*}$ and $q_{i}$ the momenta of the secondaries. The matrix element

$M_{a b}^{(n)}$ describes production of $n+2$ real final state partons and contains any number of virtual ones. $d \Phi^{(n)}$ is the full phase space factor of the ensemble of $n+2$ massless particles:

$$
d \Phi_{a b}^{(n)}\left(q_{1}, q_{2}, \ldots, q_{n}\right)=\left(d p_{a}\right)\left(d p_{b}\right) \prod_{i=1}^{n}\left(d q_{i}\right)(2 \pi)^{4} \delta^{(4)}\left(p_{a}+p_{b}+\sum_{i=1}^{n} q_{i}-Q\right),
$$

where

$$
(d q)=\frac{d^{3} \mathbf{q}}{2 \omega(2 \pi)^{3}}, \quad \omega=q_{0}=|\mathbf{q}| .
$$

The "source functions" $u$ are attached to each parton in (2.1). They help to extract an arbitrary final state observable once the functional is known. The fully inclusive measurement, that is when one allows for production of any number of particles with arbitrary momenta, corresponds to setting all $u=1$. This gives $G(Q, u=1)=1$ corresponding to normalization to the total cross section. In this section we work in the no-recoil approximation.

\subsection{Generating functional in the soft limit}

In the large- $N_{c}$ ("planar") approximation the squared matrix element can be approximated at tree level as

$$
\left|M_{a b}^{(n)}\left(q_{1}, \ldots, q_{n}\right)\right|^{2} \simeq \frac{\left(4 \pi^{2} \bar{\alpha}_{s}\right)^{n}}{n !} \sum_{\text {perm }} W_{a b}\left(q_{i_{1}}, \ldots, q_{i_{n}}\right) \cdot\left|M_{a b}^{(0)}\right|^{2} \quad \bar{\alpha}_{s}=\frac{N_{c} \alpha_{s}}{\pi},
$$

where $W_{a b}$ is the "multiple antenna" function [4] describing the production probability of $n$ soft real gluons $q_{i}$ :

$$
W_{a b}\left(q_{1}, q_{2}, \ldots, q_{n}\right)=\frac{\left(p_{a} p_{b}\right)}{\left(p_{a} q_{1}\right)\left(q_{1} q_{2}\right) \cdots\left(q_{n} p_{b}\right)} .
$$


This result holds in the soft gluon approximation $q_{i} \ll p_{a}, p_{b}$. In invariant terms,

$$
\left(q_{i} p_{a}\right),\left(q_{i} p_{b}\right) \ll\left(p_{a} p_{b}\right),
$$

or, equivalently, $\left(q_{i}, p_{a}+p_{b}\right) \ll 2\left(p_{a} p_{b}\right)=Q^{2}$ giving

$$
\omega_{i} \ll E_{a} \simeq E_{b} \simeq Q / 2
$$

with $E_{a}, E_{b}$ and $\omega_{i}$ the energies in the centre-of-mass of the "dipole" $(a b)$. In this approximation the phase space can be simplified as

$$
d \Phi_{a b}^{(n)}\left(q_{1}, \ldots, q_{n}\right) \simeq\left(d p_{a}\right)\left(d p_{b}\right)(2 \pi)^{4} \delta^{4}\left(p_{a}+p_{b}-Q\right) \cdot \prod_{i=1}^{n}\left(d q_{i}\right) \equiv d \Phi_{a b}^{(0)} \cdot \prod_{i=1}^{n}\left(d q_{i}\right) .
$$

The expression (2.3) was derived implying strong ordering of gluon energies:

$$
\omega_{i_{n}} \ll \omega_{i_{n-1}} \ll \ldots \ll \omega_{i_{2}} \ll \omega_{i_{1}} \ll Q / 2 .
$$

The answer, however, is symmetric with respect to momenta of participating gluons. Therefore, we may ignore the ordering between gluons and set a common upper bound $E=\frac{1}{2} Q$ for gluon energies.

Extracting the quarks whose momenta in the soft gluon approximation are not affected by radiation, we may write

$$
G(u ; Q) \simeq \int d \Phi_{a b}^{(0)} u\left(p_{a}\right) u\left(p_{b}\right)\left|M_{a b}^{(0)}\right|^{2} \cdot \mathcal{G}\left(p_{a}, p_{b} ; \frac{1}{2} Q\right)
$$

The functional $\mathcal{G}\left(p_{a}, p_{b} ; E\right)$ describes production of any number of gluons with energies limited from above by some value $E$, off the dipole formed by the quarks with momenta $p_{a}, p_{b}$. By setting $E=\frac{1}{2} Q$ we obtain the multi-gluon generating functional describing $e^{+} e^{-}$annihilation process with $s=Q^{2}$.

The generating functional contains proper virtual factors for each $n$-gluon contribution. Omitting for the moment the virtual corrections, we have

$$
\mathcal{G}_{(\text {real })}\left(p_{a}, p_{b} ; E\right) \equiv \sum_{n} \int W_{a b}\left(q_{1}, \ldots, q_{n}\right) \prod_{i=1}^{n}\left[\omega_{i} d \omega_{i} \frac{d \Omega}{4 \pi} \bar{\alpha}_{s} \cdot u\left(q_{i}\right) \cdot \vartheta\left(E-\omega_{i}\right)\right] .
$$

Due to the symmetric structure of the antenna function (2.3) one may choose $W_{a b}\left(q_{1} \ldots q_{n}\right)$ to represent any energy ordered ensemble of gluons and drop the symmetry factor $1 / n !$.

\subsection{Evolution equation}

To obtain an evolution equation for the generating functional we need to exploit the structure of the multi-gluon antenna distribution (2.3) and to deduce a recurrence relation. To construct a recurrence relation one should select an "evolution variable" 
to order emitted gluons. In the logic of the collinear approximation this is done by ordering the angles of successive gluon emissions, which ordering takes full care of the destructive interference contributions in the soft region $[3-5,8]$ and preserves the probabilistic parton multiplication picture. In particular, this procedure was applied to the expression (2.8) to construct the HERWIG event generator [5,15].

However, as was already stated, the distribution (2.3) is valid in the soft limit for arbitrary angles, and one is tempted to use this property in order to lift off the collinear approximation. The derivation of the evolution equation avoiding the small angle approximation was done in [11-13] where the upper bound $E$ on gluon energies in the event centre-of-mass was treated as the "evolution time" parameter. Let us recall the corresponding construction employing the energy as an evolution variable.

\subsubsection{Energy as an evolution variable}

We take the ordering in the energy of emitted gluons in the center-of-mass of the event (2.5) and deduce an evolution equation for the soft generating functional. The evolution equation follows from the exact recurrence relation - the factorization property of the multi-gluon antenna function (2.3b) — which reads

$$
W_{a b}\left(q_{1}, q_{2}, \ldots, q_{n}\right)=W_{a b}\left(q_{\ell}\right) \cdot W_{a \ell}\left(q_{1}, \ldots q_{\ell-1}\right) W_{\ell b}\left(q_{\ell+1}, \ldots, q_{n}\right)
$$

where $\ell$ is any gluon. To construct the evolution equation one takes $\ell$ to be the most energetic among the gluons.

Introducing the scaled antenna function

$$
\widehat{W}_{a b}(q) \equiv \omega^{2} \cdot W_{a b}(q)=\frac{\xi_{a b}}{\xi_{a q} \xi_{b q}}, \quad \xi_{i k}=1-\cos \Theta_{i k},
$$

which depends only on the angles between partons, we obtain

$$
E \partial_{E} \mathcal{G}\left(p_{a}, p_{b} ; E\right)=\int \frac{d \Omega}{4 \pi} \bar{\alpha}_{s} \widehat{W}_{a b}(q)\left[u(q) \mathcal{G}\left(p_{a}, q ; \omega\right) \mathcal{G}\left(q, p_{b} ; \omega\right)-\mathcal{G}\left(p_{a}, p_{b}, \omega\right)\right]_{\omega=E}
$$

Here the subtraction term takes care of virtual corrections, so that taking all $u(q)=1$ one derive the desired normalization

$$
\left.\mathcal{G}\left(p_{a}, p_{b} ; E\right)\right|_{u=1}=1
$$

No recoil was included up to now; it will be considered later.

\subsubsection{Integral evolution equation and Monte Carlo}

By exponentiating the total one-gluon emission probability we obtain the Sudakov form factor which describes the probability that the dipole does not radiate gluons with energies up to a given value $E$ :

$$
S\left(p_{a}, p_{b} ; E\right)=\exp \left\{-\int^{E} \frac{d \omega}{\omega} \frac{d \Omega}{4 \pi} \bar{\alpha}_{s}\left(q_{t}^{a b}\right) \widehat{W}_{a b}(q) \vartheta\left(q_{t}^{a b}-Q_{0}\right)\right\} .
$$


Here $q_{t}^{a b}$ is the invariant transverse momentum of the gluon with respect to the pair of quarks:

$$
\left(q_{t}^{a b}\right)^{2}=\frac{2\left(p_{a} q\right)\left(p_{b} q\right)}{\left(p_{a} p_{b}\right)}=\frac{2}{W_{a b}(q)},
$$

and an arbitrary parameter $Q_{0}$ has been introduced as a collinear cutoff. By constructing the logarithmic derivative of the Sudakov form factor over the maximal gluon energy,

$$
E \partial_{E} \ln S\left(p_{a}, p_{b} ; E\right)=-\left.\int \frac{d \Omega}{4 \pi} \bar{\alpha}_{s}\left(q_{t}^{a b}\right) \widehat{W}_{a b}(q) \vartheta\left(q_{t}^{a b}-Q_{0}\right)\right|_{\omega=E},
$$

and plugging this expression into (2.10) we can trade the virtual subtraction term for the Sudakov factor. By so doing we arrive at the equivalent equation for the soft generating functional in the integral form:

$$
\begin{aligned}
\mathcal{G}\left(p_{a}, p_{b} ; E\right)= & S\left(p_{a}, p_{b} ; E\right)+\int^{E} \frac{d \omega}{\omega} \int \frac{d \Omega}{4 \pi} u(q) \cdot \frac{S\left(p_{a}, p_{b} ; E\right)}{S\left(p_{a}, p_{b} ; \omega\right)} \\
& \cdot \bar{\alpha}_{s}\left(q_{t}^{a b}\right) \widehat{W}_{a b}(q) \vartheta\left(q_{t}^{a b}-Q_{0}\right) \cdot \mathcal{G}\left(p_{a}, q ; \omega\right) \mathcal{G}\left(q, p_{b} ; \omega\right) .
\end{aligned}
$$

Iteration of this equation can be interpreted as parton branching which can be realised as a Monte Carlo (Markov) process. To see this, observe that the iteration kernel

$$
d \mathcal{P}\left(p_{a}, p_{b}, q ; E\right)=\frac{d \omega}{\omega} \frac{d \Omega}{4 \pi} \frac{S\left(p_{a}, p_{b} ; E\right)}{S\left(p_{a}, p_{b} ; \omega\right)} \cdot \bar{\alpha}_{s}\left(q_{t}^{a b}\right) \widehat{W}_{a b}(q) \vartheta\left(q_{t}^{a b}-Q_{0}\right)
$$

can be written as the product of two probability distributions (here the boundary $q_{t}^{a b}>Q_{0}$ is implicit):

$$
\begin{aligned}
& d \mathcal{P}\left(p_{a}, p_{b}, q ; E\right)=d \mathcal{P}_{a b}^{(1)}(\omega, E) \cdot d \mathcal{P}_{a b}^{(2)}\left(\Omega_{q}\right), \\
& \mathcal{P}_{a b}^{(1)}(\omega, E)=\frac{S\left(p_{a}, p_{b} ; E\right)}{S\left(p_{a}, p_{b} ; \omega\right)}, \quad d \mathcal{P}_{a b}^{(2)}\left(\Omega_{q}\right)=\bar{\alpha}_{s} \frac{d \Omega_{q}}{4 \pi} \frac{\xi_{a b}}{\xi_{a q} \xi_{q b}}\left(\int \bar{\alpha}_{s} \frac{d \Omega}{4 \pi} \frac{\xi_{a b}}{\xi_{a q} \xi_{q b}}\right)^{-1} .
\end{aligned}
$$

In the Monte Carlo process one tries to generate emission of a gluon with momentum $q$ off a given dipole. The first distribution, $d \mathcal{P}^{(1)}$, provides the gluon energy $\omega$, if the transverse momentum bound is satisfied, and the second one, $d \mathcal{P}^{(2)}$, its direction $\Omega_{q}$. If the boundary is not satisfied, the dipole does not emit. One has

$$
\int d \mathcal{P}\left(p_{a}, p_{b}, q, E\right)=1-S\left(p_{a}, p_{b} ; E\right)
$$

which shows that the Sudakov factor gives the probability of not emitting a soft gluon within the resolution $q_{t}^{a b}>Q_{0}$. 


\subsection{Mean multiplicity}

By construction the generating functional (2.15) embeds only soft gluon radiation. Incorporating double logarithmic (simultaneously soft- and collinear-enhanced) contributions, it also contains single logarithmic effects due to emission of soft gluons at large angles.

Here we demonstrate an application of the dipole-based evolution to the calculation of subleading large-angle soft gluon corrections. In the collinear approach, such corrections are treated as due to "multi-jet" configurations (contributing to the "coefficient function") rather than jet evolution ("anomalous dimension"), see [8].

Consider mean gluon multiplicity. In order to obtain an equation for the multiplicity of secondary partons, one applies to (2.10) the variational derivative over the probing function $u(k)$ and integrates over $k$, while setting $u \equiv 1$ for all remaining probing functions (one-particle inclusive measurement):

$$
E \partial_{E} N\left(\xi_{a b}, E\right)=\int \frac{d \Omega}{4 \pi} \bar{\alpha}_{s}\left(k_{t}^{a b}\right) \widehat{W}_{a b}(q)\left[1+N\left(\xi_{q a}, E\right)+N\left(\xi_{q b}, E\right)-N\left(\xi_{a b}, E\right)\right]_{\omega=E} .
$$

Introducing $\mathcal{N}=1+N$ we have [16]

$$
\mathcal{N}^{\prime}\left(\xi_{a b}\right)=E \partial_{E} \mathcal{N}\left(\xi_{a b}\right)=\int \frac{d \Omega}{4 \pi} \bar{\alpha}_{s}\left(k_{t}^{a b}\right) \widehat{W}_{a b}(q)\left[\mathcal{N}\left(\xi_{q a}\right)+\mathcal{N}\left(\xi_{q b}\right)-\mathcal{N}\left(\xi_{a b}\right)\right]
$$

In order to illustrate the difference with the standard approach based on the angular ordering we will analyse (2.20) in the double logarithmic approximation which neglects hard parton splittings and recoil effects. For the time being we will ignore the running of the coupling; the corresponding corrections will be addressed later.

Representing the integrand on the r.h.s. of $(2.20)$ as

$\frac{\xi}{\xi_{1} \xi_{2}}\left[\mathcal{N}\left(\xi_{1}\right)+\mathcal{N}\left(\xi_{2}\right)-\mathcal{N}(\xi)\right]=\frac{1}{\xi_{2}}\left[\frac{\xi}{\xi_{1}} \mathcal{N}\left(\xi_{1}\right)-\mathcal{N}(\xi)\right]+\frac{1}{\xi_{1}}\left[\frac{\xi}{\xi_{2}} \mathcal{N}\left(\xi_{2}\right)-\mathcal{N}(\xi)\right]-\frac{\xi-\xi_{1}-\xi_{2}}{\xi_{1} \xi_{2}} \mathcal{N}(\xi)$

and using the $1 \leftrightarrow 2$ symmetry, we obtain

$$
\mathcal{N}^{\prime}(\xi)=\int \frac{d \Omega}{4 \pi} \bar{\alpha}_{s}\left\{\frac{2}{\xi_{2}}\left[\frac{\xi}{\xi_{1}} \mathcal{N}\left(\xi_{1}\right)-\mathcal{N}(\xi)\right]-\frac{\xi-\xi_{1}-\xi_{2}}{\xi_{1} \xi_{2}} \mathcal{N}(\xi)\right\} .
$$

We can perform the integration over the azimuth of $\mathbf{q}$ around the direction of $\mathbf{p}_{a}$,

$$
\int \frac{d \Omega}{4 \pi}=\frac{1}{2} \int_{0}^{2} d \xi_{1} \cdot \int \frac{d \phi_{1}}{2 \pi}
$$

Using the relation

$$
\left\langle\frac{1}{\xi_{2}}\right\rangle \equiv \int \frac{d \phi_{1}}{2 \pi} \frac{1}{\xi_{2}}=\frac{1}{\left|\xi-\xi_{1}\right|}
$$


we get

$$
\left\langle\frac{\xi-\xi_{1}-\xi_{2}}{\xi_{1} \xi_{2}}\right\rangle=\frac{1}{\xi_{1}}\left(\frac{\xi-\xi_{1}}{\left|\xi-\xi_{1}\right|}-1\right)=-\frac{2}{\xi_{1}} \vartheta\left(\xi_{1}-\xi\right)
$$

As a result,

$$
\mathcal{N}^{\prime}(\xi)=\bar{\alpha}_{s} \int_{0}^{2} \frac{d \xi_{1}}{\left|\xi-\xi_{1}\right|}\left[\frac{\xi}{\xi_{1}} \mathcal{N}\left(\xi_{1}\right)-\mathcal{N}(\xi)\right]+\bar{\alpha}_{s} \int_{\xi}^{2} \frac{d \xi_{1}}{\xi_{1}} \mathcal{N}(\xi),
$$

with the $E$ dependence implicit. The first contribution we split into integrals over small and large angles, $0 \leq \xi_{1} \leq \xi$ and $\xi<\xi_{1} \leq 2$. Introducing the integration variable $\eta=\xi_{1} / \xi \leq 1$ in the first region and $\eta=\xi / \xi_{1}$ in the second, $\frac{1}{2} \xi \leq \eta \leq 1$, the equation (2.23) takes the form [16]

$$
\mathcal{N}^{\prime}(\xi)=\int_{0}^{1} \frac{d \eta \bar{\alpha}_{s}}{1-\eta}\left[\frac{1}{\eta} \mathcal{N}(\eta \xi)-\mathcal{N}(\xi)\right]+\int_{\frac{1}{2} \xi}^{1} \frac{d \eta \bar{\alpha}_{s}}{1-\eta}\left[\mathcal{N}\left(\eta^{-1} \xi\right)-\mathcal{N}(\xi)\right]
$$

This result we represent as a sum of two terms,

$$
E \partial_{E} \mathcal{N}(\xi)=\int_{0}^{1} \frac{d \eta \bar{\alpha}_{s}}{\eta} \mathcal{N}(\eta \xi)+\Delta(\xi)
$$

where

$$
\Delta(\xi)=\int_{0}^{1} \frac{d \eta \bar{\alpha}_{s}}{1-\eta}[\mathcal{N}(\eta \xi)-\mathcal{N}(\xi)]+\int_{\frac{1}{2} \xi}^{1} \frac{d \eta \bar{\alpha}_{s}}{1-\eta}\left[\mathcal{N}\left(\eta^{-1} \xi\right)-\mathcal{N}(\xi)\right] .
$$

The first integral term on the r.h.s. of (2.25a) generates the standard DL anomalous dimension for the mean multiplicity; the second one constitutes a subleading correction.

\subsubsection{The main term}

We have to solve the equation

$$
E \partial_{E} \mathcal{N}^{\mathrm{DL}}(\xi ; E)=\int_{0}^{1} \frac{d \eta \bar{\alpha}_{s}}{\eta} \mathcal{N}^{\mathrm{DL}}(\eta \xi ; E)
$$

with the initial condition

$$
\left.\mathcal{N}(\xi ; E)\right|_{E \sqrt{2 \xi}=Q_{0}}=1 .
$$

The solution is a function of a single variable $Q_{\xi} \equiv E \sqrt{2 \xi}$ (the maximal transverse momentum of partons radiated by the dipole).

The argument of the running coupling in (2.26) can be approximated as

$$
\bar{\alpha}_{s}\left(k_{t}^{a b}\right)=\bar{\alpha}_{s}\left(E \sqrt{\frac{2 \xi_{1} \xi_{2}}{\xi}}\right) \simeq \bar{\alpha}_{s}\left(E \sqrt{2 \xi_{1}}\right) .
$$


The correction to this approximation turns out to be negligible since the logarithmic factor $\ln \left(\xi_{2} / \xi\right)$ vanishes in the collinear limit $\xi_{1} \rightarrow 0$ :

$$
\propto \int \frac{d \xi_{1}}{\xi_{1}}\left(\beta_{0} \bar{\alpha}_{s}^{2}\right) \cdot \ln \frac{\xi_{2}}{\xi}=\mathcal{O}\left(\bar{\alpha}_{s}^{2}\right) .
$$

Thus we obtain the double differential equation

$$
\left(\frac{d^{2}}{d \ln Q_{\xi}}\right)^{2} \mathcal{N}^{\mathrm{DL}}\left(Q_{\xi}\right)=2 \bar{\alpha}_{s}\left(Q_{\xi}\right) \mathcal{N}^{\mathrm{DL}}\left(Q_{\xi}\right), \quad Q_{\xi}=E \sqrt{2 \xi},
$$

with the initial conditions $\mathcal{N}\left(Q_{0}\right)=1$ and $\mathcal{N}^{\prime}\left(Q_{0}\right)=0$. Its solution for the fixed coupling case reads

$$
\mathcal{N}^{\mathrm{DL}}\left(Q_{\xi}\right)=\cosh \left(\gamma \ln \frac{Q_{\xi}}{Q_{0}}\right) \simeq \frac{1}{2}\left(\frac{Q_{\xi}}{Q_{0}}\right)^{\gamma}
$$

where the anomalous dimension $\gamma$ is given the correct expression

$$
\gamma=\bar{\alpha}_{s} \int_{0}^{1} \frac{d \eta}{\eta} \cdot \eta^{\frac{1}{2} \gamma}=\bar{\alpha}_{s} \cdot \frac{2}{\gamma} \Longrightarrow \gamma=\sqrt{2 \bar{\alpha}_{s}} .
$$

\subsubsection{The correction term}

Let us analyse the correction term (2.25b) keeping the contributions of the order of $\bar{\alpha}_{s}^{3 / 2}$ while neglecting contributions $\mathcal{O}\left(\bar{\alpha}_{s}^{2}\right)$. The answer depends on the value of the opening angle $\xi$.

Small opening angles $(\xi \ll 1)$. It is the region of small opening angles that gives a dominant contribution to the integral determining the DL anomalous dimension in (2.26). In this entire region the correction (2.25b) does not contribute at the $\bar{\alpha}_{s}^{3 / 2}$ level. Indeed, substituting the solution (2.28) into the expression (2.25b) and setting $\xi \rightarrow 0$ in the lower limit of the second integral we get

$$
\begin{aligned}
\frac{\Delta(\xi ; E)}{\mathcal{N}(\xi ; E)} & =\bar{\alpha}_{s} \int_{0}^{1} \frac{d \eta}{1-\eta}\left[\eta^{\gamma / 2}+\eta^{-\gamma / 2}-2\right] \\
& =\bar{\alpha}_{s} \cdot\left[2 \psi(1)-\psi\left(1+\frac{1}{2} \gamma\right)-\psi\left(1-\frac{1}{2} \gamma\right)\right]=\bar{\alpha}_{s} \cdot \frac{1}{4} \gamma^{2} \psi^{\prime \prime}(1)+\ldots \simeq \frac{1}{2} \bar{\alpha}_{s}^{2} \psi^{\prime \prime}(1) .
\end{aligned}
$$

Large opening angle $(\xi \simeq 2)$. At the same time, $\Delta$ contributes to the multiplicity of the "fully open" dipole, $\xi=2$. The expression (2.25b) for $\Delta(2 ; E)$ contains one integral:

$$
\Delta(2 ; E)=\bar{\alpha}_{s} \int_{0}^{1} \frac{d \eta}{1-\eta}[\mathcal{N}(\eta \cdot 2 ; E)-\mathcal{N}(2 ; E)] .
$$

Expanding the difference of the multiplicity factors,

$$
\mathcal{N}(\eta \cdot 2 ; E)-\mathcal{N}(2 ; E)=E \partial_{E} \mathcal{N}(2 ; E) \cdot \frac{1}{2} \ln \eta+\ldots,
$$


we derive

$$
\Delta(2 ; E) \simeq E \partial_{E} \mathcal{N}(2 ; E) \cdot \bar{\alpha}_{s} \int_{0}^{1} \frac{d \eta}{1-\eta} \cdot \frac{1}{2} \ln \eta=-\frac{\pi^{2}}{12} \bar{\alpha}_{s} \cdot E \partial_{E} \mathcal{N}(2 ; E)
$$

Integrating this correction over energy we finally obtain an order $\alpha_{s}$ correction to the dipole multiplicity due to emission of two soft energy ordered gluons:

$$
\mathcal{N}(2 ; E)=\mathcal{N}^{\mathrm{DL}}(2 ; E) \cdot\left(1-\frac{\pi^{2}}{12} \bar{\alpha}_{s}+\ldots\right)
$$

In the standard approach, this correction belongs to the coefficient function and originates from a "four-jet" configuration with two large-angle energy ordered soft gluons as additional "jets" [8].

\subsubsection{Other observables sensitive to large-angle gluon effects}

There is a variety of observables that are sensitive to soft gluon radiation at large angles. Among them one can mention production of heavy quark pairs in jets [16], nonglobal jet observables [11] including various particle distributions and correlations related with particle flows in the inter-jet regions [12,13,17-21]. The dipole-based evolution equation (2.15) is well suited for taking into account single logarithmic correction effects due to multiple soft gluons in these observables and elsewhere.

At the same time, it remains insufficient for building a realistic Monte Carlo event generator which is impossible without incorporating full parton decay probabilities, including hard parton splittings, and of the recoil effects to ensure energy-momentum conservation.

\section{Attempt to include recoil in dipole multiplication}

Generally speaking, a gluon emitted by a dipole cannot be ascribed as offspring to either of the two partons that form the dipole. However, in the collinear limit when the gluon momentum $q$ is quasi-parallel to one of the two hard partons, say, $p_{a}$, it is the parton $a$ that can be said to "independently split" into two, sharing its momentum, $P_{a} \rightarrow p_{a}+q$. A small change in the "spectator" momentum $P_{b} \rightarrow p_{b}$ which is necessary to compensate for the virtuality of the $(a, q)$ pair, vanishes in the collinear limit. A general recoil strategy has to be formulated in such a way that in the collinear limit the answer reduces to the standard DGLAP parton splitting function $P_{a} \rightarrow p_{a}+q$ that includes hard momentum configurations, $p_{a} \sim q$. This is a source of a significant single-logarithmic correction, this time not from soft gluon radiation at large angles but from hard collinear-enhanced emission.

Thus, in order to properly formulate a recoil strategy, one must split the soft

dipole radiation function $W_{a b}(q)$ into two pieces, $W_{a b}^{(a)}(q)$ and $W_{a b}^{(b)}(q)$, each of which 
incorporates the collinear singularity when $\mathbf{q} \| \mathbf{p}_{a}$ or $\mathbf{q} \| \mathbf{p}_{b}$, respectively:

$$
W_{a b}(q)=\frac{\left(p_{a} p_{b}\right)}{\left(p_{a} q\right)\left(q p_{b}\right)}=W_{a b}^{(a)}(q)+W_{a b}^{(b)}(q),
$$

or, graphically,
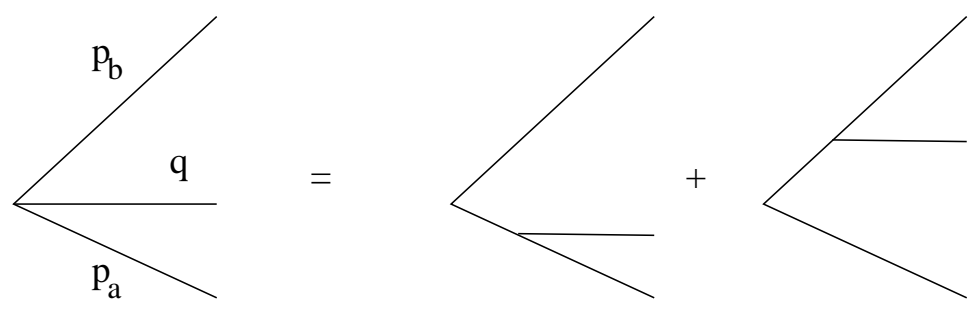

One can do this differently, for example by introducing [14]

$$
W_{a b}^{(a)}(q)=\frac{\left(p_{a} p_{b}\right)}{\left(p_{a} q\right)\left(p_{a}+p_{b}, q\right)}, \quad W_{a b}^{(b)}(q)=\frac{\left(p_{a} p_{b}\right)}{\left(p_{b} q\right)\left(p_{a}+p_{b}, q\right)} .
$$

Another possibility to split the dipole is given by the "conditional probabilities" $[5,6]$

$$
W_{a b}^{(a)}(q)=W_{a b}(q) \cdot \frac{1}{2}\left[1+\frac{\left(q p_{a}\right)\left(p_{b} Q\right)-\left(q p_{b}\right)\left(p_{a} Q\right)}{\left(p_{a} p_{b}\right)(q Q)}\right]=\frac{1}{2 \omega^{2} \xi_{q a}}\left[1+\frac{\xi_{a b}-\xi_{q a}}{\xi_{q b}}\right] .
$$

The distribution (3.3) produces the exact angular ordering upon averaging over the azimuthal angle $\phi$ of the gluon momentum $\mathbf{q}$ around the singular direction $\mathbf{p}_{a}$ :

$$
\left\langle W_{a b}^{(a)}\right\rangle_{\phi_{\mathbf{q}, \mathbf{p} a}}=\frac{1}{\omega^{2} \xi_{q a}} \cdot \vartheta\left(\xi_{a b}-\xi_{a q}\right) .
$$

Note that in all cases the distributions $W_{a b}^{(c)}(q)(c=a, b)$ fall fast and essentially become irrelevant when the emission angle exceeds the opening angle of the parent dipole, $\xi_{q c}>\xi_{a b}$, that is away from the angular ordered kinematics.

\subsection{Recoil strategy}

Consider the elementary process

$$
P_{a}+P_{b} \Longrightarrow p_{a}+p_{b}+q .
$$

One ascribes definite recoil pattern separately to the two terms in (3.1). Thus, for the first term $W_{a b}^{(a)}$ which is collinear singular in the direction $\mathbf{q} \| \mathbf{p}_{a}$, we choose, following the Catani-Seymour prescription [14]

$$
p_{b}^{(a)}=(1-y) P_{b} .
$$


It defines the two light-like vectors $P_{a}, P_{b}$ that represent the momenta of the parent partons prior to the gluon emission:

$$
\begin{aligned}
p_{a}^{(a)} & =z P_{a}+(1-z) y P_{b}-k_{t}, \\
q & =(1-z) P_{a}+z y P_{b}+k_{t},
\end{aligned}
$$

where $k_{t}$ is ortogonal to $P_{a}$ and $P_{b}$. The on-mass-shell condition $p_{a}^{2}=0$ gives

$$
\left(P_{a}+y P_{b}-q\right)^{2}=0 \quad \Longrightarrow \quad y=\frac{\left(q P_{a}\right)}{\left(P_{a}-q, P_{b}\right)}
$$

The light-cone fraction $z$ of the parent momentum $P_{a}$ carried by the final quark $p_{a}$ is given by the expression

$$
1-z=\frac{\left(q P_{a}\right)}{\left(P_{a} P_{b}\right)}
$$

In the collinear limit $y \rightarrow 0$. In the soft limit both $y \rightarrow 0$ and $z \rightarrow 1$.

We now recast the phase space element in terms of the "parent momenta":

$$
\begin{aligned}
d \Phi_{a b}(q) & =\left(d p_{a}\right)\left(d p_{b}\right)(d q)(2 \pi)^{4} \delta^{(4)}\left(p_{a}+p_{b}+q-Q\right) \\
& =\left(d P_{a}\right)\left(d P_{b}\right)(2 \pi)^{4} \delta^{(4)}\left(P_{a}+P_{b}-Q\right) \cdot(d q) \times J_{a b}^{(a)}(q) \equiv d \Phi_{a b}^{(0)} \cdot(d q) J_{a b}^{(a)}(q) .
\end{aligned}
$$

The Jacobian of this transformation reads

$$
J_{a b}^{(a)}(q)=\left(1-\frac{\left(q P_{a}\right)}{\left(P_{a}-q, P_{b}\right)}\right)\left(1-\frac{\left(q P_{b}\right)}{\left(P_{a} P_{b}\right)}\right)^{-1}=\frac{1-y}{z} .
$$

The gluon radiation probability we represent as a sum of two contributions:

$$
d \Phi_{a b}(q) \cdot W_{a b}(q) \Rightarrow d \Phi_{a b}^{(0)} \cdot(d q)\left[W_{a b}^{(a)}(q) J_{a b}^{(a)}(q)+W_{a b}^{(b)}(q) J_{a b}^{(b)}(q)\right] .
$$

Finally, the evolution equation in the differential form becomes

$$
\begin{aligned}
& E \partial_{E} \mathcal{G}\left(P_{a}, P_{b} ; E\right)=\int \frac{d \Omega}{4 \pi} \bar{\alpha}_{s}\left(q_{t}^{a b}\right) \vartheta\left(q_{t}^{a b}-Q_{0}\right) \\
& \times \sum_{c=a, b} J_{a b}^{(c)}(q) \widehat{W}_{a b}^{(c)}(q)\left[u(q) \mathcal{G}\left(p_{a}^{(c)}, q ; E\right) \mathcal{G}\left(q, p_{b}^{(c)} ; E\right)-\mathcal{G}\left(P_{a}, P_{b} ; E\right)\right]_{E=\omega} .
\end{aligned}
$$

In the graphic form it can be represented as



This evolution equation is the modification of (2.10) that includes recoil as described above. The gluon radiation function $\widehat{W}_{a b}(q)=\omega^{2} W_{a b}(q)$ is split according to (3.9) 
into the two pieces which are collinear singular when $q$ is parallel to $p_{a}$ or to $p_{b}$, correspondingly, see [14]. The energy of the hardest real gluon $q_{0} \equiv \omega=E$ plays the rôle of the evolution parameter.

By introducing the Sudakov form factors this equation can be recast in an integral form as it has been done in the soft case in (2.15).

$$
\begin{aligned}
\mathcal{G}\left(P_{a}, P_{b} ; E\right)= & S\left(P_{a}, P_{b} ; E\right)+\int^{E} \frac{d \omega}{\omega} \int \frac{d \Omega}{4 \pi} u(q) \cdot \frac{S\left(P_{a}, P_{b} ; E\right)}{S\left(P_{a}, P_{b} ; \omega\right)} \vartheta\left(q_{t}^{a b}-Q_{0}\right) \\
& \times \bar{\alpha}_{s}\left(q_{t}^{a b}\right) \sum_{c=a, b} J_{a b}^{(c)}(q) \widehat{W}_{a b}^{(c)}(q) \cdot \mathcal{G}\left(p_{a}^{(c)}, q ; \omega\right) \mathcal{G}\left(q, p_{b}^{(c)} ; \omega\right) .
\end{aligned}
$$

This equation generalizes (2.15) by taking into account the energy-momentum recoil and again satisfies the normalization $\mathcal{G}\left(P_{A}, P_{b} ; E\right)=1$ for all $u(q)=1$ (cf. (2.11)).

An iterative solution of this integral equation defines a Monte Carlo process for generating multi-parton ensembles with account of collinear non-enhanced singlelogarithmic corrections due to large-angle soft gluon emission.

Now we are going to check if the dipole recoil scheme described above is consistent with known analytical results concerning the collinear resummation. The simplest observable of this type is an inclusive energy distribution of the final-state quark.

\subsection{The non-singlet quark energy distribution}

The non-singlet quark fragmentation function is obtained by taking the derivative of the generating functional $G_{a b}$ with respect to the quark source $u\left(p_{a}\right)$ and setting all the remaining source functions $u=1$. This way we obtain the distribution in the momentum of the quark $a$ accompanied by any number of gluons. If the quark energy is taken large, $(1-x) \ll 1$, all radiated gluons are soft and the analysis simplifies significantly. The problem becomes essentially Abelian ${ }^{1}$ and is described by multiple independent radiation of soft gluons by the quark.

\subsubsection{Collinear approximation}

We shall restrict ourselves to configurations in which all radiated gluons have small emission angles with respect to the quark direction. This - quasi-collinear - approximation is sufficient for the analysis of the anomalous dimension which accumulates collinear ("mass") singularities of the fragmentation function in all orders and describes the scaling violation.

In this kinematics the measured variable $x$ is given by

$$
x=1-\sum_{i} y_{i}, \quad \omega_{i}=y_{i} E, \quad p_{a} \simeq x P_{a} .
$$

\footnotetext{
${ }^{1}$ Non-Abelian effects due to final state cascading of gluons reduce to making the effective coupling in the radiation probability of a primary gluon $k$ run with $k_{\perp}^{2}$.
} 
What matters is the total energy $1-x$ carried, on average, by an ensemble of radiated soft gluons.

Looking for collinear singular contributions - the terms of the type $\bar{\alpha}_{s} \ln (1-$ $x) \cdot \ln Q_{0}$ - we may omit the two last terms on the r.h.s. of the equation (3.10b) that contain the factor $W_{a b}^{(b)}(q)$ which is non-singular when the gluon momentum $\mathbf{q}$ becomes collinear to $\mathbf{p}_{a}$.

One is then left with contributions involving only the singular antenna piece $W_{a b}^{(a)}(q)$ :

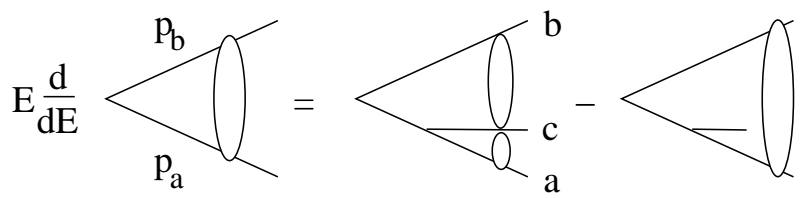

Since our observable is inclusive, we may use unitarity arguments to simplify the equation. Indeed, by the nature of the adopted recoil strategy, production of gluons by the "upper" dipole $(b c)$ in (3.13) does not affect the momentum of the quark $a$. Therefore, since such emissions do not affect the measurement, they are subject to real-virtual cancellation and can be neglected.

One is left to consider only gluons emitted in the lower blob involving the parton $a$ :



This equation generates multiple gluon emissions off the quark.

By construction, successive emissions are ordered in gluon energies. And here comes the crucial observation: as we have discussed above, the emission angle of the gluon $c$ is essentially limited from above by the aperture of the parent dipole, $\xi_{a b}$, see (3.4). Therefore, soft gluons generated by the evolution equation (3.14) turn out to be ordered, simultaneously, in energies and angles with respect to the radiating quark. Instead we know that the DGLA equation is obtaine from ordering only in collinear variables, disregarding the relative energies of emitted partons. The corresponding equation in the present dipole-formulation looks as follows.

\subsubsection{Evolution equation with dipole recoil strategy}

Since the radiated gluons are soft, we use the distribution $W_{a b}^{(a)}(q)$ defined in (3.3) which describes the soft part of the gluon radiation probability (splitting function). We may also approximately set $J_{a b}^{(a)}(q)=1$.

In going from the generating functional $\mathcal{G}\left(P_{a}, P_{b} ; E\right)$ in (3.10) to the single particle distribution, we express the latter as a function of the angle between the partons $\xi_{a b}$, the energy scale $E \simeq E_{a}$ and the energy fraction of the triggered quark: $D\left(\xi_{a b}, E, x\right)$. 
Performing integration over the azimuthal angle $\phi_{a q}$ (see (3.4)) one deduces from (3.10)

$$
\begin{aligned}
D(\xi ; E ; x)=\delta(1-x)+ & \int^{1} \frac{d y}{y} \int^{\xi} \frac{d \xi^{\prime}}{2 \xi^{\prime}} \bar{\alpha}_{s} \vartheta\left(y E \sqrt{\xi^{\prime}}-Q_{0}\right) \\
& \times\left[D\left(\xi^{\prime}, y E, \frac{x}{1-y}\right)-D\left(\xi^{\prime}, y E, x\right)\right],
\end{aligned}
$$

where $\xi=\xi_{a b}$ is the angular aperture of the original dipole $(a b)$ while $\xi^{\prime}=\xi_{a c}$ is the aperture of the secondary $(a c)$-dipole, and for the sake of simplicity we have restricted ourselves to the soft part of the gluon radiation probability, $d y / y$. Equivalently one may write ${ }^{2}$

$$
\begin{aligned}
& D(\xi ; E ; x)=\delta(1-x) S(E \sqrt{\xi}) \\
& +\int^{1} \frac{d y}{y} \int^{\xi} \frac{d \xi^{\prime}}{2 \xi^{\prime}} \bar{\alpha}_{s} \vartheta\left(y E \sqrt{\xi^{\prime}}-Q_{0}\right) \frac{S(E \sqrt{\xi})}{S(y E \sqrt{\xi})} D\left(\xi^{\prime}, y E, \frac{x}{1-y}\right),
\end{aligned}
$$

with the Sudakov form factor given by the expression

$$
\ln S(E \sqrt{\xi})=-\int^{1} \frac{d y}{y} \int^{\xi} \frac{d \xi^{\prime}}{2 \xi^{\prime}} \bar{\alpha}_{s} \vartheta\left(y E \sqrt{\xi^{\prime}}-Q_{0}\right) .
$$

The distribution $D$ describing real gluon emission in the integrands of (3.15) is evaluated at $\xi^{\prime}$ since a gluon produced by the dipole $(a c)$ has an angle with respect to $a$ effectively bounded from above by $\xi_{a c}$, see (3.4).

It is worth noticing that going from the equation for the generating functional (3.10) to that for the inclusive quark spectrum, we have replaced the full angle aperture $\xi=\xi_{a b}$ by the opening angle $\xi^{\prime}$ of the (ac) dipole in the virtual (subtraction) term as well. In so doing we followed the preceding observation according to which virtual radiation of gluons constituting the "wider" dipole $(b c)$ had been already cancelled by corresponding real emissions, without affecting the energy of the triggered quark.

Equations (3.15) correctly satisfy the sum rule for the first moment: integrating over $x$ one obtains 1 for the number of quarks a (to see this one uses (2.18)). However, they are in conflict with the Poisson nature of the multiple soft gluon radiation and contradict the collinear resummation result leading to the DGLAP equation.

\subsubsection{Analysis of the equation}

Introducing the transverse momentum scale variable $Q=E \sqrt{\xi}$ and the corresponding integration variable $q=\omega \sqrt{\xi^{\prime}}$ we get

$$
D(x, Q)=\delta(1-x)+\int_{Q_{0} / Q}^{1} \frac{d y}{y} \int_{Q_{0}}^{y Q} \frac{d q}{q} \bar{\alpha}_{s}\left[D\left(\frac{x}{1-y}, q\right)-D(x, q)\right],
$$

\footnotetext{
${ }^{2}$ The $y$ integration in (3.15b) is actually limited from above by $y=1-x$ since the distribution $D(\xi, Q, z) \propto \Theta(1-z)$
} 
or, in terms of the standard Mellin moment representation,

$$
D_{N}(Q)=1+\int_{Q_{0} / Q}^{1} \frac{d y}{y} \int_{Q_{0}}^{y Q} \frac{d q}{q} \bar{\alpha}_{s}\left[(1-y)^{N}-1\right] D_{N}(q) .
$$

In the region of large $x$, such that $1-x \ll 1$ (but $1-x \gg Q_{0} / Q$ ), the essential Mellin moments are large, $N \sim(1-x)^{-1}$, and cut from below the $y$-integral:

$$
D_{N}(Q) \simeq 1-\int_{1 / N}^{1} \frac{d y}{y} \int_{Q_{0}}^{y Q} \frac{d q}{q} \bar{\alpha}_{s} D_{N}(q) .
$$

By iterating this equation one obtains an oscillating double logarithmic series in $\ln Q$ and $\ln N$,

$$
D_{N}^{(n)}(Q) \sim \frac{\left(-\bar{\alpha}_{s} \ln N \ln Q\right)^{n}}{(n !)^{2}}+\ldots
$$

Here one combinatorial factor $1 / n$ ! comes from the kinematical ordering of gluon energies, and the second $1 / n$ ! from the ordering of the angles with respect to the quark of the successively radiated gluons. This series sums up into a Bessel function instead of an exponent in $\ln Q$ with the well known DGLAP "anomalous dimension"

$$
D_{N}^{(n)}(Q) \sim \frac{\left(-\bar{\alpha}_{s} \ln N \ln Q\right)^{n}}{n !}, \quad D_{N}(Q) \propto Q^{-\bar{\alpha}_{s} \ln N}
$$

where we have neglected for the sake of simplicity the running of the coupling.

In general (whether the coupling runs or not), the anomalous dimension $\gamma_{N}$ is defined as

$$
\gamma_{N}\left(\bar{\alpha}_{s}(Q)\right)=\frac{d}{d \ln Q} \ln D_{N}(Q)
$$

For $\bar{\alpha}_{s}=$ const this gives

$$
D_{N}(Q)=\left(\frac{Q}{Q_{0}}\right)^{\gamma_{N}\left(\bar{\alpha}_{s}\right)} .
$$

Substituting this ansatz into (3.19) and evaluating the logarithmic derivative, we obtain an equation for the new anomalous dimension:

$$
\gamma_{N}\left(\bar{\alpha}_{s}\right) \simeq-\bar{\alpha}_{s} \int_{1 / N}^{1} d y y^{\gamma_{N}-1}=-\bar{\alpha}_{s} \frac{1-\exp \left(-\gamma_{N} \ln N\right)}{\gamma_{N}}
$$

Perturbatively, when $\bar{\alpha}_{s} \ln ^{2} N \ll 1$, we have the correct one loop expression

$$
\gamma_{N}\left(\bar{\alpha}_{s}\right) \simeq-\bar{\alpha}_{s} \ln N+\mathcal{O}\left(\bar{\alpha}_{s}^{2} \ln ^{3} N\right)
$$

while in higher loops it starts to deviate significantly from the DGLAP answer. In general, introducing the variable $w$

$$
\gamma_{N}=-\frac{w}{\ln N}
$$


the anomalous dimension is given by the solution of the equation

$$
\bar{\alpha}_{s} \ln ^{2} N=\frac{w^{2}}{e^{w}-1} .
$$

The r.h.s. hits the maximum at certain $w=w_{0}$, so that in the region of large moments $N$, starting from $\bar{\alpha}_{s} \ln ^{2} N=$ const, the anomalous dimension becomes complex valued and the distribution starts to oscillate.

The origin of the failure of the energy ordering strategy can be understood already at the level of two emitted gluons.

\subsubsection{Two gluon emission}

Consider the emission of two soft gluons $p_{1}, p_{2}$ off the parton $q$ of the $q \bar{q}$ dipole. The antenna functions that potentially contribute in the collinear limit (recall that we keep all gluon angles with respect to the quark $a=q$ to be small) are displayed here:

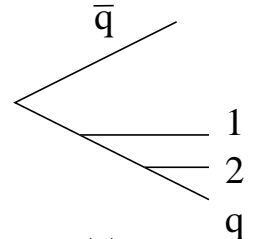

(a)

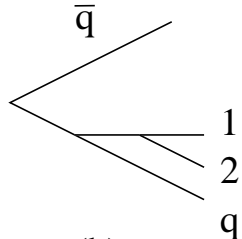

(b)

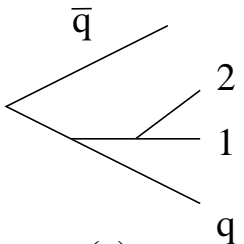

(c)

The first two graphs correspond to the splitting of the dipole $(a 1)$ :

$$
W_{a 1}^{(a)}(2) \rightarrow \vartheta\left(\xi_{a 1}-\xi_{a 2}\right) \quad \text { and } \quad W_{a 1}^{(1)}(2) \rightarrow \vartheta\left(\xi_{a 1}-\xi_{12}\right),
$$

while the third one,

$$
W_{1 \bar{q}}^{(1)}(2) \rightarrow \vartheta\left(\xi_{1 \bar{q}}-\xi_{12}\right),
$$

is the relevant part of the large-aperture dipole $(1 \bar{q})$. Due to the local recoil prescription used, only the contribution $(a)$ affects the momentum of the quark $q$. In the two remaining ones, (b) and (c), the gluon 2 borrows its energy-momentum from the gluon 1 and does not produce any quark recoil. Therefore, these contributions cancel against corresponding virtual corrections in the inclusive quark measurement. In conclusion, within the adopted recoil strategy, only the graph (a) should be kept, and we obtain the following phase space for the two-gluon emission:

$$
\xi_{a 2} \ll \xi_{a 1} \ll \xi_{a \bar{q}} \quad \text { and } \quad \omega_{2}<\omega_{1} \ll E \text {. }
$$

The first condition comes from the angular ordering in the graph (3.23)(a), and the second condition - from the energy ordering of successive emissions.

We know, however, that in order to obtain the DGLAP equation that properly resums collinear singular contributions, one needs to assemble angular (or, transverse momentum) ordered emissions (the first ordering), regardless to the order of 
gluon energies (the second one). At the same time, the dipole logic is leading us to the double-ordered gluon ensemble, according to (3.24). As a result, instead of a simple Poisson distribution of soft radiation $(1 / n !)$ we obtain something like a Bessel function distribution $\left(1 /(n !)^{2}\right)$.

What is missing here is actually the coherence of QCD radiation. As well known, a soft gluon $\omega_{2}$, with $\omega_{2} \ll \omega_{1} \ll E_{a}$, could be emitted at large angles $\left(\xi_{a 2} \gg \xi_{a 1}\right)$ directly by the original parton $q+p_{1} \simeq q$. In the language of Feynman amplitudes, such radiation occurs as a coherent sum of the graphs (b) and (c).

Another way to discuss this point is the following. The dipole radiation pattern of soft gluon 2 off the composite antenna $(a 1 \bar{q})$ can be represented as follows:

$$
W_{a 1}(2)+W_{1 \bar{q}}(2)=\left[W_{a 1}(2)+W_{1 \bar{q}}(2)-W_{a \bar{q}}(2)\right]+W_{a \bar{q}}(2) .
$$

Beyond the large- $N_{c}$ approximation, the latter term (corresponding to emission of the gluon 2 by the quark-antiquark dipole) acquires a $N_{c}^{2}$-suppressed correction, $N_{c} \rightarrow N_{c}-1 / N_{c}:$

$$
\frac{N_{c}}{2}\left(W_{a 1}(2)+W_{1 \bar{q}}(2)\right) \Longrightarrow \frac{N_{c}}{2} \cdot\left[W_{a 1}(2)+W_{1 \bar{q}}(2)-W_{a \bar{q}}(2)\right]+C_{F} \cdot W_{a \bar{q}}(2) .
$$

This is in line with the collinear factorization theorem. Indeed, the factor in square brackets is non-singular when $\mathbf{p}_{2}$ becomes parallel to the direction of the quark or antiquark momentum so that these collinear enhancements are contained by the quark dipole term $W_{a \bar{q}}(2)$ and must be proportional to the "colour charge" (Casimir operator) $C_{F}$ of the quark.

The only collinear singularity of the first contribution on the r.h.s. is that of $\mathbf{p}_{2}$ parallel to $\mathbf{p}_{1}$ which suggest an interpretation of this term as independent emission of the gluon 2 by the previous generation (harder) gluon 1. The full two soft gluon emission probability takes the form

$$
d w(1,2) \propto C_{F} \frac{N_{c}}{2} \cdot W_{a \bar{q}}(1)\left[W_{a 1}(2)+W_{1 \bar{q}}(2)-W_{a \bar{q}}(2)\right]+C_{F}^{2} \cdot W_{a \bar{q}}(1) W_{a \bar{q}}(2) .
$$

In the large- $N_{c}$ limit, this is identical to the original dipole expression. At the same time, the representation (3.26) suggests a different recoil strategy when it comes to incorporating the energy-momentum conservation.

The first term in (3.26) describes cascade gluon multiplication: $q \bar{q} \rightarrow 1$ followed by $1 \rightarrow 2$. In the inclusive quark distribution this is subject to the real-virtual cancellation. Energy ordered configurations, $\omega_{2} \ll \omega_{1}$ cancel leaving no trace; "hard" gluon splittings with $\omega_{2} \simeq \omega_{1}$ modify one gluon emission by giving rise to $\alpha_{s}\left(k_{1 \perp}^{2}\right)$ for the radiation intensity of the primary gluon 1 by the quark. 
The second term describes independent radiation of two gluons off the quark — the second order term of the Poisson distribution of primary gluons — which is necessary to correctly recover the DGLAP quark fragmentation function.

Equation (3.26) provides a probabilistic representation for the two-gluon production in terms of a combination of independent and cascade gluon emissions. With account of hard parton splittings and of the natural energy recoil prescription, it served as the base for constructing the HERWIG parton event generator $[5,15]$. The corresponding probabilistic representation for production of three and four energy ordered gluons was developed in [6] and described in [8], which resulted in a formulation of the parton multiplication scheme based on the exact angular ordering of successive parton splittings.

The difference between the two strategies becomes transparent. In the dipole scheme, successive gluons borrowing the energy from the quark were ordered in emission angles,

$$
W_{a \bar{q}}^{(a)}(1) W_{a 1}^{(a)}(2), \quad \xi_{2 a}<\xi_{1 a}<1,
$$

while in (3.26) the angle of the softest gluon is not bounded, and the two emissions are totally independent,

$$
W_{a \bar{q}}^{(a)}(1) W_{a \bar{q}}^{(a)}(2), \quad \xi_{2 a}<1, \quad \xi_{1 a}<1 .
$$

Let us see how this has happened, from the point of view of the gluon-gluon splitting.

In the quasi-collinear configuration, $\xi_{a 1} \ll 1$, the radiation pattern of the softest gluon 2 consists of a "narrow" and a "wide-angle" dipoles, $(a 1)$ and $(1 \bar{q})$. When, within the dipole recoil strategy, the "wide-angle" antenna $W_{1 \bar{q}}(2)$ is split into the pieces collinear singular with respect to the directions of the first generation gluon (1) and of the antiquark,

$$
W_{1 \bar{q}}(2)=W_{1 \bar{q}}^{(1)}(2)+W_{1 \bar{q}}^{(\bar{q})}(2),
$$

the contribution $W_{1 \bar{q}}^{(1)}(2)$ is looked upon as radiation of the gluon 2 by the gluon 1 , whichever the kinematical configuration of the gluon 1 with respect to its parent quark (dipole factorization). At the same time, within the HERWIG logic (collinear factorization), the gluon-gluon multiplication occurs only in a restricted angular aperture, $\xi_{21}<\xi_{1 a}$. Indeed, in the kinematical region $\xi_{1 a} \ll \xi_{21} \ll 1$ the two last terms in the combination

$$
\left[W_{a 1}(2)+W_{1 \bar{q}}(2)-W_{a \bar{q}}(2)\right]
$$

on the r.h.s. of (3.25) cancel:

$$
\lim _{\xi_{1 a} \rightarrow 0} \xi_{1 a}^{2} \cdot\left[W_{1 \bar{q}}(2)-W_{a \bar{q}}(2)\right]=\mathcal{O}\left(\frac{\xi_{1 a}}{\xi_{12}}\right) \rightarrow 0
$$


(while the first antenna term, $W_{a 1}(2)$, is restricted in angle on its own).

Physically, the gluon 2 radiated off the gluon 1 in the "wide" dipole (3.23) (c) at relatively large angles, $\xi_{21}>\xi_{1 a}$, is produced by the grandparent parton a (quark). Correspondingly, it must borrow energy from the quark rather than locally from the dipole $(1 \bar{q})$ to which it belongs from the point of view of the colour connection topology.

Reformulating this way the local dipole recoil prescription modifies the energy ordered evolution equation. The modification reduces to substituting the full dipole opening angle $\xi=\xi_{a b}$ for the running angle parameter $\xi^{\prime}$ in the argument of the distribution $D$ on the r.h.s. of (3.15). As demonstrated in the Appendix, this way one recovers the approximate DGLAP equation for the quark fragmentation function.

\section{Conclusions}

Monte Carlo generation of QCD events is a quarter century old business, based on the structure of resummation of collinear enhanced Feynman diagram contributions. The probabilistic parton cascade picture was first established for one-particle inclusive quantities (DGLAP evolution equations for DIS structure functions and $e^{+} e^{-}$ fragmentation functions, [1]), generalised via "jet-calculus" [2] and, with account of the soft gluon coherence $[3-5,8]$, has laid the base for probabilistic description of the internal structure of parton jets and their ensembles.

The corresponding coherent branching Monte Carlo schemes (HERWIG, in particular) reproduce correctly both the leading double logarithmic effects (LL) in various QCD observables as well as subleading single logarithmic collinear-enhanced terms. One may recall as examples NLL corrections to the mean particle multiplicity [22] and to the inclusive particle spectra from light parton $[7,23]$ and heavy quark initiated jets [24], to global event shapes in $e^{+} e^{-}$annihilation [25], as well as many other quantities sensitive to parton cascades.

Collinear-non-enhanced ("large angle") soft gluon radiation provides significant NNLL corrections to global event characteristics (e.g., mean particle multiplicity); it is also responsible for inter-jet multiplicity and energy flows and determines the structure of various non-global observables [11-13]. Effects of multiple soft gluon radiation at large angles lie beyond the scope of the standard (collinear) approach

and must be treated order by order in perturbation theory (while collinear enhanced contributions are resummed in all orders).

An elegant expression [4] for the multiple soft gluon production probability (2.3) is valid for arbitrary angles and offers a possibility of improving the parton picture. The structure of multi-gluon distribution (2.3) naturally suggests an interpretation in terms of a chain of colour connected dipoles. By choosing an "evolution time" variable this chain may be generated via a Markov process of successive dipole splittings. An evolution time can in principle be chosen differently. We have used energy ordering of 
gluons for this purpose. The generating functional that we have constructed with the help of the corresponding evolution equation allows one to calculate specific effects due to multiple emission of soft gluons at large angles in the large- $N_{c}$ approximation.

In order to construct a realistic Monte Carlo generator for multi-parton ensembles it is imperative, however, to formulate an adequate recoil prescription which would ensure energy-momentum conservation at every successive step of the parton (dipole) multiplication.

In the present paper we addressed the question, whether the "dipole factorization" extends beyond the no-recoil approximation. In other words, whether the splitting of a colour dipole into two can be treated independently of the prehistory of the system, that is locally in the evolution time (which is a necessary condition for constructing a Markov process).

Having taken an inclusive energy distribution of the final quark as the simplest example of a collinear sensitive observable, we have shown that a naive implementation of the dipole recoil strategy results in violating the collinear factorization.

However, the existing Monte Carlo implementations of the dipole picture (for recent examples see for instance [26-28]) chose to order successive gluon emissions in (dipole centre of mass) transverse momenta rather than in (laboratory frame) gluon energies. Such a construction apparently prevents the problem under consideration from appearing in the leading collinear approximation, that is in all orders in the QCD coupling corresponding to the one-loop level in the anomalous dimension.

Indeed, as we have seen above, the problem with the quark recoil appears when a softer gluon (2) is emitted at a large angle than its predecessor (1). The inclusive quark energy distribution is sensitive to the region where the two gluons have comparable energies. Then, a softer one that carries a slightly smaller energy but is radiated at a large angle, acquires a larger transverse momentum, and therefore, by construction, is looked upon as "the first" by the $k_{\perp}$-ordered MC scheme. Therefore, "the second" gluon in such a picture, that is the one with smaller transverse momentum, is automatically having a smaller emission angle; in the leading logarithmic approximation the coherence is respected.

The problem of an interplay between energy-momentum recoil and soft gluon coherence discussed in this paper gets postponed but should be kept in mind in view of attempts at constructing the next-to-leading order QCD MC generators.

\section{Acknowledgements}

We thank the organisers and participants of the CERN TH jet phenomenology seminar for the opportunity to extensively discuss the issues raised in the paper. We are especially grateful to Walter Giele, Gösta Gustafson, David Kosower, Leif Lönnblad, Zoltan Nagy, Gavin Salam, Jim Samuelsson, Mike Seymour, Torbjorn Sjöstrand, Peter Skands, Davidson Soper and Bryan Webber for illuminating discussions. 


\section{A. Global recoil and correspondence with DGLAP}

We have no universal recipe under sleeve for extending the dipole gluon multiplication picture beyond the no-recoil approximation. However, for a simple example of the inclusive quark distribution discussed in Section 3 the situation is straightforward to cure.

As we saw from the previous discussion, the origin of the failure lay in the fact that the softest gluon emitted at angles larger than the angle of the previous branchings did not contribute to the quark recoil. So, it suffices, by brute force, to permit the gluons of all generations to contribute to the quark recoil, irrespectively to the value of their emission angles.

This amounts to replacing in the integrand of $(3.15 \mathrm{~b})$ the distribution $D$ evaluated at $\xi=\xi_{a c}$ with the distribution evaluated at the full opening angle $\bar{\xi}=\xi_{a b}$. One then has the different equation,

$$
\begin{aligned}
D(\bar{\xi}, E ; x) & =\delta(1-x) S(E \sqrt{\bar{\xi}}) \\
& +\int^{1} \frac{d y}{y} \int^{\bar{\xi}} \frac{d \xi}{\xi} \frac{\bar{\alpha}_{s}}{2} \vartheta\left(y E \sqrt{\xi}-Q_{0}\right) \frac{S(E \sqrt{\bar{\xi}})}{S(y E \sqrt{\bar{\xi}})} D\left(\bar{\xi}, y E ; \frac{x}{1-y}\right)
\end{aligned}
$$

which actually corresponds to the DGLAP evolution. To see this, we iterate equation (A.1) to obtain the series

$$
\begin{aligned}
& D(\bar{\xi}, E ; x)=S(E \sqrt{\bar{\xi}}) \\
& \times\left\{\delta(1-x)+\sum_{n=1}^{\infty} \prod_{i}\left(\int^{1} \frac{d y_{i}}{y_{i}} \int^{\bar{\xi}} \frac{d \xi_{i}}{\xi_{i}} \frac{\bar{\alpha}_{s}}{2} \theta\left(y_{i} E \sqrt{\xi_{i}}-Q_{0}\right)\right) \cdot \delta\left(1-\frac{x}{1-\sum y_{i}}\right) \Theta_{\text {en-ord. }}\right\},
\end{aligned}
$$

where

$$
\Theta_{\text {en-ord }}=\Theta\left(y_{n}<\cdots<y_{1}<1\right)
$$

is the product of with theta-functions which ensures the energy ordering of gluons. Now, due to the symmetry of the multiple integral (A.2) with respect to energy and angular variables, we can replace the energy ordering with the angular ordering:

$$
\Theta_{\text {en-ord }} \Longrightarrow \Theta_{\text {ang-ord }}=\Theta\left(\xi_{n}<\cdots<\xi_{1}<\bar{\xi}\right) \text {. }
$$

Now the gluons are no longer ordered in energies (the gluon $q_{i}$ does not need to be softer then $q_{i-1}$ ) but in the emission angle with respect to the quark. In the angular ordered form, a soft gluon radiated at a relatively large angle can be said to be emitted before any other (harder or softer) gluons that move in a collinear bunch around the quark. This is in accord with the colour coherence according to which a large-angle soft gluon "sees" only the total colour charge of a collinear group of partons. 
To derive the DGLAP equation we replace

$$
\frac{d \omega_{i}}{\omega_{i}}=\frac{d y_{i}}{y_{i}}=\frac{d z_{i}}{1-z_{i}}, \quad 1-\sum y_{i}=z_{1} \cdots z_{n}
$$

where now $1-z_{i}$ is the local fraction of energy taken away by the gluon $q_{i}$ from the parent parton (quark), while $y_{i}$ is the gluon energy fraction with respect to $E$, the energy of the primary parton $P_{a}$ :

$$
y_{i}=\left(1-z_{i}\right) z_{i-1} \cdots z_{1}
$$

We finally obtain the evolution equation

$$
\begin{aligned}
D(Q, x) & =\delta(1-x) S(Q)+\int_{Q_{0}^{2}}^{Q^{2}} \frac{d q_{t}^{2}}{q_{t}^{2}} \int_{0}^{1-\frac{Q_{0}}{q_{t}}} d z \frac{\bar{\alpha}_{s}\left(q_{t}\right)}{2(1-z)} \cdot \frac{S(Q)}{S\left(q_{t}\right)} D\left(q_{t}, \frac{x}{z}\right) \\
& =\delta(1-x)+\int_{Q_{0}^{2}}^{Q^{2}} \frac{d q_{t}^{2}}{q_{t}^{2}} \int_{0}^{1-\frac{Q_{0}}{q_{t}}} d z \frac{\bar{\alpha}_{s}\left(q_{t}\right)}{2(1-z)} \cdot\left(D\left(q_{t}, \frac{x}{z}\right)-D\left(q_{t}, x\right)\right)
\end{aligned}
$$

with $Q=E \sqrt{\xi}$ and $q_{t}=y E \sqrt{\xi}$. The upper boundary of the $z$-integration, $1-Q_{0} / q_{t}$, can be replaced by 1 in the logarithmic collinear approximation, which results in the integral equation that correctly gives (the soft piece of) the DGLAP anomalous dimension.

Finally, let us mention another obstacle that one faces when trying to construct the full realistic Monte Carlo scheme starting from the energy ordered gluon ensembles.

In order to match the full DGLAP anomalous dimension, one has to include the "hard part" of the splitting function by adding to the distributions $\widehat{W}_{a b}^{(c)}(q)$ the pieces vanishing at $\omega=0$. In the equation (A.6) this calls for the replacement

$$
\frac{d y_{i}}{y_{i}}=\frac{d z_{i}}{1-z_{i}} \quad \Longrightarrow \quad d z_{i}\left(\frac{1}{1-z_{i}}-\frac{1}{2}\left(1+z_{i}\right)\right) \text {. }
$$

However, while we could cast the leading soft distribution in (A.1) in terms of the local energy fraction, $d y_{i} / y_{i}=d z_{i} /\left(1-z_{i}\right)$, the finite term $\left(1+z_{i}\right) d z_{i}$ cannot be expressed locally, via the single variable $y_{i}$, see (A.5).

\section{References}

[1] V.N. Gribov, L.N. Lipatov, Sov.J.Nucl.Phys. 15 (1972) 438;

G. Altarelli and G. Parisi, Nucl. Phys. B126 (1977) 298;

Yu.L. Dokshitzer, Sov. Phys. JETP 46 (1977) 641.

[2] K. Konishi, A. Ukawa and G. Veneziano, Nucl. Phys. B157 (1979) 45. 
[3] A.H. Mueller, Phys. Lett. 104B (1981) 161;

B.I. Ermolayev and V.S. Fadin, JETP Lett. 33 (1981) 285;

A. Bassetto, M. Ciafaloni, G. Marchesini and A. H. Mueller, Nucl. Phys. B 207 (1982) 189; Yu.L. Dokshitzer, V.S. Fadin and V.A. Khoze, Zeit. Phys. C15 (1982) 325; ibid C18 (1983) 37.

[4] A. Bassetto, M. Ciafaloni and G. Marchesini, Phys. Rept. 100 (1983) 201.

[5] G. Marchesini and B.R. Webber, Nucl. Phys. B238 (1984) 1.

[6] Yu.L. Dokshitzer and S.I. Troian, "Asymptotic Freedom and Local Parton-Hadron Duality", in: Proceedings of the XIX LNPI Winter School, vol. 1, p. 144, Leningrad, 1984.

[7] Ya.I. Azimov, Yu.L. Dokshitzer, V.A. Khoze and S.I. Troian, Zeit. Phys. C27 (1985) 65.

[8] Yu.L. Dokshitzer, V.A. Khoze, A.H. Mueller and S.I. Troian, Basics of Perturbative QCD, Editions Frontières, Gif-sur-Yvette, 1991.

[9] G. Gustafson, Phys. Lett. B175 (1986).

[10] Yu.L. Dokshitzer, V.A. Khoze and S.I. Troian, Sov. J. Nucl. Phys. 50 (1989) 505 [Yad. Fiz. 50 (1989) 808].

[11] M. Dasgupta and G.P. Salam, Phys. Lett. B512 (2001) 323 [hep-ph/0104277].

[12] M. Dasgupta and G.P. Salam, JHEP 0203 (2002) 017 [hep-ph/0203009];

A. Banfi, G. Marchesini and G. Smye, JHEP 0208 (2002) $006 \quad$ [hep-ph/0206076];

R.B. Appleby and M.H. Seymour, JHEP 0212 (2002) 063 [hep-ph/0211426];

C.F. Berger, T. Kucs and G. Sterman, Phys. Rev. D65 (2002) 094031

[hep-ph/0110004];

C.F. Berger, T. Kucs and G. Sterman, Phys. Rev. D68 (2003) 014012

[hep-ph/0303051].

[13] Yu.L. Dokshitzer and G. Marchesini, JHEP 0303 (2003) 040 [hep-ph/0303101].

[14] S. Catani and M. Seymour, Nucl. Phys. B485 (1997) 291, Erratum-ibid. B510 (1998) 503

[hep-ph/9605323].

[15] G. Marchesini and B. R. Webber, Nucl. Phys. B310 (1988) 461.

[16] G. Marchesini and A.H. Mueller, Phys. Lett. B575 (2003) 37 [hep-ph/0308284];

G. Marchesini and E. Onofri, JHEP 0407 (2004) $031 . \quad$ [hep-ph/0404242]

[17] A. Banfi, G. Marchesini and G. Smye, JHEP 0208 (2002) 006. [hep-ph/0206076]

[18] N. Kidonakis and G. Sterman, Phys. Lett. B387 (1996) 867, Nucl. Phys. B505 (1997) 321; N. Kidonakis, G. Oderda and G. Sterman, Nucl. Phys. B531 (1998) 365;

E. Laenen, G. Oderda and G. Sterman, Phys. Lett. B438 (1998) 173. 
[19] Yu.L. Dokshitzer, V.A. Khoze, G. Marchesini and B.R. Webber, Phys. Lett. B245 (1990) 243.

[20] Ya.I. Azimov, Yu.L. Dokshitzer, V.A. Khoze and S.I. Troian, Phys. Lett. 165B (1985) 147.

[21] G. Marchesini and B.R. Webber, Phys. Rev. D38 (1988) 3419.

[22] A.H. Mueller, Nucl. Phys. B213 (1983) 85.

[23] S. Catani, B.R. Webber and G. Marchesini, Nucl. Phys. B349 (1991) 635;

S. Gieseke, P. Stephens and B.R. Webber, JHEP 0312 (2003) 045. [hep-ph/0310083]

[24] Yu.L. Dokshitzer, V.A. Khoze and S.I. Troian, Phys. Rev. D53 (1996) 89; [hep-ph/9506425]

M. Cacciari and S. Catani, Nucl. Phys. B617 (2001) $253 . \quad$ [hep-ph/0107138]

[25] S. Catani, L. Trentadue, G. Turnock and B.R. Webber, Nucl. Phys. B407 (1993) 3.

[26] Leif Lonnblad, Comput. Phys. Commun. 71 (1992) 15 [hep-ph/0010017].

[27] Z. Nagy and D.E. Soper, JHEP 0803 (2008) $030 . \quad$ [arXiv:0801.1917] Z. Nagy and D.E. Soper, JHEP 0807 (2008) $025 . \quad$ [arXiv:0805.0216]

[28] W.T. Giele, D.A. Kosower and P.Z. Skands, Phys. Rev. D78 (2008) 014026. [arXiv:0707.3652] 